\title{
High Rate and High Temperature Fracture Behaviour of Polycrystalline Diamond
}

\author{
M. Petrovic ${ }^{1, a}$, A. Ivankovic ${ }^{1, b}$, N. Murphy ${ }^{1, c}$ \\ ${ }^{1}$ UCD School of Electrical, Electronic and Mechanical Engineering \\ University College Dublin, Dublin 4, Ireland \\ amarin.petrovic@ucd.ie, balojz.ivankovic@ucd.ie, neal.murphy@ucd.ie
}

Keywords: PCD, Fracture Toughness, Young's Modulus, Loading Rate, Temperature, Microstructure

\begin{abstract}
Polycrystalline diamond (PCD) materials have a variety of applications, mainly as cutting tools for machining non-ferrous metals and non-metallic materials. A significant application of PCD is in oil and gas industry for rock drilling operations. Other important areas, such as mining, have yet to reach their full potential. These cutters/tools are subjected to high operating temperatures, impact loads and abrasive wear during these operations, which may lead to their sudden failure. An advantage of these materials is that their structure and composition can be engineered to return properties required for specific applications and operations.
\end{abstract}

In this study, the mechanical and fracture properties of two grades of PCD have been determined at dynamic loading rates up to $5 \mathrm{~m} / \mathrm{s}$ and elevated temperature up to $600{ }^{\circ} \mathrm{C}$ in laboratory conditions using three point bending (TPB) and split-Hopkinson pressure bar apparatus (SHPB). The results show Young's modulus to be completely rate insensitive, while fracture toughness changes significantly. Two dominant regions can be observed on the rate scale: one predominated by the conductivity of the material and the other predominated by the rate of fracture where binder degrades locally at the crack tip. This behaviour was related to the material microstructure, where similarities and differences in the fracture of two different grades of PCD are shown examining polished fractured specimens using scanning electron microscopy, revealing mechanisms of fracture over the range of loading rates.

\section{Introduction}

Polycrystalline diamond is a sintered mass of randomly oriented, intergrown, diamond particles in the presence of cobalt as a catalyst, where the sintering process is achieved in high pressure and high temperature conditions. Premature failure of the cutting tool made of PCD, caused by tool fracture and chipping, is a frequent problem in the drilling industry. Considering the expenses of one day's drilling, changing the drill bit in the middle of the drilling process costs hundreds of thousands of euro. Therefore the most important demand on the cutting tool is to resist fracture. Having said that, the main aim for the manufacturers of the drilling bits is to understand the fracture process of these materials and try to improve their fracture properties. In this work, the effects of dynamic loading rates and high temperatures are considered.

\section{Materials and Methods}

In this study, high temperature testing chambers, a laboratory scale three point bend test rig and a splitHopkinson pressure bar apparatus have been designed and used for testing the fracture and mechanical properties of two different grades of PCD: G6 grade constituted of $6 \mu \mathrm{m}$ average grain size and G30 grade constituted of $30 \mu \mathrm{m}$ average grain size. Standard fracture and material analysis were then carried out on each sample to determine its apparent fracture toughness $K_{I b}$ and Young's modulus $E$. 
Young's modulus tests were performed on miniature size disk shaped PCD specimens of 9mm diameter and $3 \mathrm{~mm}$ thickness at three different strain rates and at $300^{\circ} \mathrm{C}$ in air. Small, custom built, cylindrical heating unit was made of stainless steel and attached to the SHPB setup. Heating unit has a hole on top for easy inserting and removal of the test pieces. Two cartridge heaters with power rating of 200W each are connected in series and inserted into holes drilled and reamed on one side of the heating unit.

Normal procedure when testing the Young's modulus at SHPB is to use Kolsky analysis [1], where the reflected and transmitted strain signals are recorded by strain gauges placed onto the incident and transmitter bars, respectively. However, the problems encountered during testing necessitated modifications of conventional Kolsky analysis. The reason for this is the very high impedance of PCD material which effectively means that the reflected wave from the steel bar is very small and lost in the noise. This required instrumenting the specimen with a minute strain gauge and measuring the strain directly rather than the conventional method of integrating the reflected strain in the incident bar. Therefore, the strain was recorded by use of strain gauges connected to a Vishay 2310B Signal Conditioning Amplifier and the $100 \mathrm{MHz}$ Handyscope HS3.

Elevated temperature tests with the SHPB method pose a problem if temperature gradients exist along the bar length because the propagation of elastic waves is affected through the effect of temperature on Young's modulus and wave velocity of the bar material. These gradients have two effects on the strain signal: a continuous change of amplitude and a continuous change of wave velocity. Correction factor is derived [2] and employed in the following form:

$$
\frac{\varepsilon_{0}}{\varepsilon_{T}}=\left(1+C_{a}\right)^{3 / 4}, \quad \frac{t_{T}}{t_{0}}=\sqrt{\frac{E_{0}}{E_{T}}}\left(1+C_{a}\right)^{3 / 4}, \quad C_{a}=\frac{a_{2}}{a_{1}}\left(T-T_{0}\right),
$$

where $T_{0}$ is ambient temperature,

$T$ is temperature of the specimen,

$a_{1}, a_{2}$ are constants that satisfy relation for linearly temperature dependent Young's modulus

$$
E=a_{1}+a_{2}\left(T-T_{0}\right) .
$$

Testing at temperatures above $300^{\circ} \mathrm{C}$ is not feasible at present, due to absence of the appropriate miniature strain gauge pattern on the market.

Fracture toughness tests were performed in three-point bending on PCD Single Edge V-notched Bend (SEVNB) specimens with machined notch of $1.24 \mathrm{~mm}$ in depth and $150 \mu \mathrm{m}$ notch root radius, using an instrumented Zwick-Roel drop-weight tower. The fracture toughness of PCD was evaluated at a temperature of $600^{\circ} \mathrm{C}$ and 3 different dynamic loading rates: $0.3 \mathrm{~m} / \mathrm{s}, 1 \mathrm{~m} / \mathrm{s}$ and $5 \mathrm{~m} / \mathrm{s}$. The load at initiation method was used in the analysis, as previously explained in [3,4], which is sufficiently accurate even for the dynamic tests as the specimens were instrumented with the minute strain gauges close to the crack tip from which the load was extracted. Strain gauges used were of MicroMeasurements type ZC-NC-G1262-120, which are free filament kanthal alloy gauges bonded by ceramic adhesive and with the measurement range of up to $1150^{\circ} \mathrm{C}$. 


\section{Results}

Dynamic Young's modulus results are presented in Figures 1a and 1b for G6 and G30 material, respectively, and compared with previously published quasistatic results [3]. At least three repeats at each combination of rate and temperature were conducted.

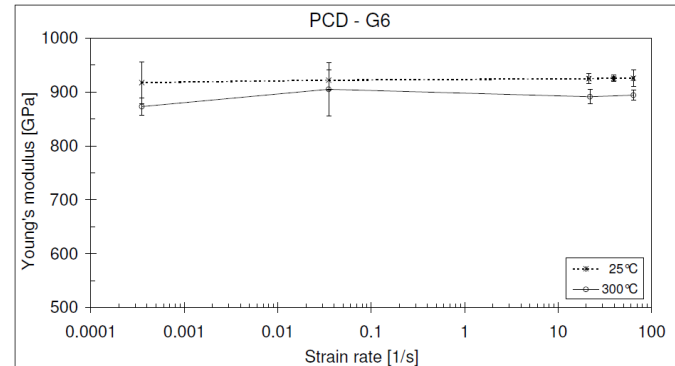

(a)

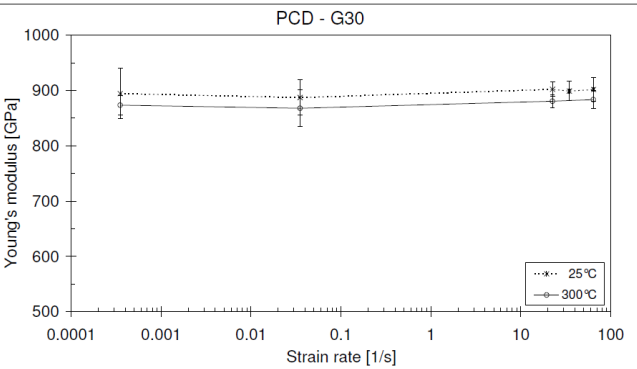

(b)

Figure 1: Young's modulus of $G 6($ (a) and $G 30$ (b) as a function of strain rate and temperature

Apparent fracture toughness results determined in three-point bending are shown in Figures $2 \mathrm{a}$ and $2 \mathrm{~b}$ for G6 and G30 material, respectively, and compared with previously published low temperature results [3].

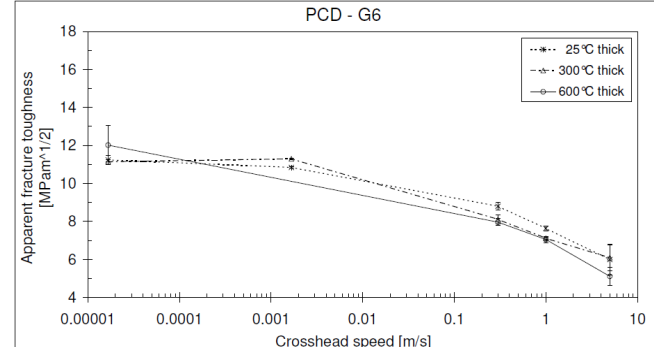

(a)

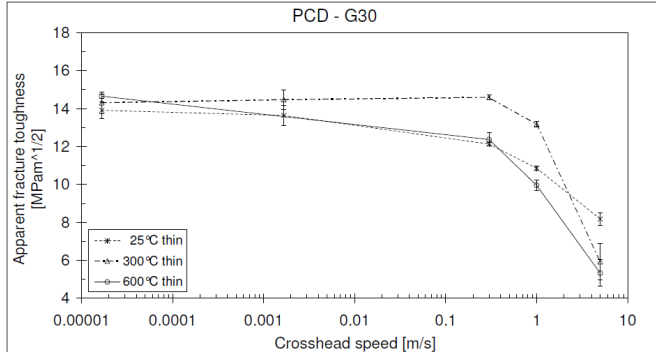

(b)

Figure 2: Apparent fracture toughness of G6 (a) and G30 (b) as a function of crosshead speed and temperature

Thick and thin samples refer to two different production quality materials and can not be compared between themselves. However, relative change in fracture toughness can be compared as both grades have the same notch root radius.

\section{Microscopy Analysis}

In order to be able to get a better insight into the fracture process, eight G6 specimens and eight G30 specimens were sectioned and polished and these polished surfaces were examined by FEG-SEM. Using energy dispersive X-ray analysis (EDX), it was found that light greyish areas represent cobalt, while dark grey corresponds to diamond grains. Comparison between sectioned surfaces of one G6 specimen fractured at quasistatic rate and another fractured at dynamic rate is shown in Figure 3. Examined regions are very close to the initial crack tip, while the fracture surface is on the left on these 
images. It can be seen that the binder in regions close to the edge is considerably deteriorated during dynamic fracture which extends up to $30 \mu \mathrm{m}$ off the fracture surface, as completely opposed to the fracture at quasistatic rate where the binder left undamaged.

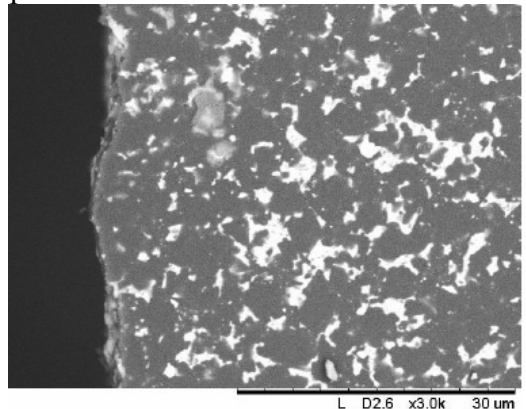

(a)

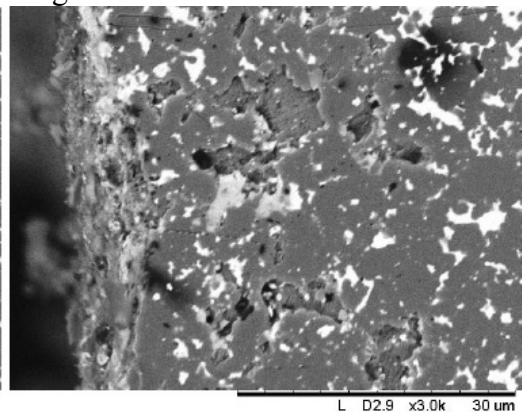

(b)

Figure 3: SEM images of the polished surface of G6 samples fractured at $1 \mathrm{~mm} / \mathrm{min}(\mathrm{a})$ and $5 \mathrm{~m} / \mathrm{s}(\mathrm{b})$

\section{Discussion and Conclusions}

It has been shown that Young's modulus of both grades of PCD exhibits no rate sensitivity across five decades of the strain rate. Slight but consistent decrease of Young's modulus with increase of the temperature is also found at high strain rates. G30 grade appears to be still less susceptible to the temperature effects, due to higher average grain size and smaller cobalt content. When testing fracture toughness, two dominant regions can be observed on the rate scale: isothermal governed by the conductivity of the material and adiabatic governed by the rate of fracture where binder degrades locally at the crack tip. Isothermal region is longer for the coarser grain material due to higher conductivity. Apparent fracture toughness shows increasing behaviour with increase in the temperature at quasistatic rates, while this is reversed at dynamic rates. Microscopy analysis discloses the region of strong deterioration of cobalt at dynamic rates. It appears that binder gets damaged before the crack even reaches it by the temperature increase ahead of the crack tip. Considering huge difference between thermal expansion of diamond and cobalt, little change in temperature causes considerable change in expansions of these two materials, inducing shear locally in ligament of PCD binder between the grains. The stress intensity factor acting at the crack tip may be reduced due to these thermal stresses, thus causing microcracks and fracture to occur at lower load levels.

The undertaken study leads to improvement of understanding of PCD behaviour and thereafter allows recommendations to be made for the improved design of the material itself and of the corresponding manufacturing processes.

\section{References:}

[1] H. Kolsky. Stress waves in solids. Clarendon Press, Oxford, 1953

[2] U.S. Lindholm, L.M. Yeakley: Experimental Mechanics Vol. 8 (1968), p. 1-9

[3] M. Petrovic, D. Carolan, A. Ivankovic, N. Murphy: Key Engineering Materials Vol. 452 (2011), p. $153-156$

[4] M. Petrovic, D. Carolan, V. Kanyanta, A. Ivankovic, Proceedings of the $6^{\text {th }}$ ICCSM (2009)

Acknowledgements: This work is funded by Enterprise Ireland and Element 6 Ltd., Shannon, Ireland 